\title{
Caractérisation du fonctionnement hydrosédimentaire de l'estuaire de la Vilaine à partir d'études bathymétriques et altimétriques (Altus)
}

\author{
Evelyne GOUBERT ${ }^{1}$, Emmanuel FRENOD ${ }^{1}$, \\ Pierre PEETERS ${ }^{2}$, Pauline THUILLIER ${ }^{2}$, Hans Jacob VESTED ${ }^{3}$, \\ Nathalie BERNARD ${ }^{4}$, Véronique VERON ${ }^{4}$, Lionel ALLANO $^{5}$ \\ ${ }^{1}$ Université de Bretagne Sud - LabSTICC, Campus Tohannic, 56000 Vannes. \\ evelyne.goubert@univ-ubs.fr,emmanuel.frenod@univ-ubs.fr \\ ${ }^{2}$ DHI 2/4 rue Edouard Nignon, CS 47202, 44372 NANTES Cedex 3, France. \\ ppe@dhigroup.com,pth@dhigroup.com \\ ${ }^{3}$ DHI Agern Allé 5, DK-2970 Hørsholm, Denmark.hvj@dhigroup.com \\ ${ }^{4}$ Institution d'Aménagement de la Vilaine, Boulevard de Bretagne - BP 11, 56 \\ 130 La Roche Bernard.nathalie.bernard@lavilaine.com \\ ${ }^{5}$ Université de Rennes 1, Station biologique de Bailleron, 56000 St Armel. \\ Lionel.allano@univ-rennes1.fr
}

\section{Résumé :}

Depuis la construction du barrage d'Arzal entre 1965 et 1970, l'estuaire de la Vilaine est soumis à un fort envasement. A partir de données bathymétriques, hydrologiques et altimétriques (Altus), il est possible d'identifier des climats hydrodynamiques à différentes échelles spatiales et temporelles et de mieux quantifier les phases d'érosion ou de dépôts associées. De plus, il a été possible de montrer l'impact d'un modelé en seillons/banquettes ou d'un modelé plan sur la dynamique de la vasière.

\begin{abstract}
:
From the construction of the Arzal's dam in 1960's, the Vilaine estuary has been rapidly in-filled. According to bathymetrical, hydrological and altimetrical (Altus) data, different hydrodynamical patterns have been identified at various spatial and temporal scales leading to erosional or depositional periods. The hydrodynamical pattern's series allows a better understanding and quantification of sedimentological processes at long term and at short term. Finally, we discussed the influence of bedforms (ridge and runnels or planar bed) on sedimentological processes over an intertidal mudflat.
\end{abstract}

Mots-clés : Vasière estuarienne intertidale, hydrodynamisme, seillons/banquettes. 


\section{Introduction}

La dynamique de la sédimentation fine en domaine estuarien est un problème récurrent depuis de nombreuses années pour les usagers des estuaires. Afin de mieux gérer ces environnements, de nombreux modèles numériques ont été conçus pour apporter des solutions d'aménagement. Pour que ces modèles soient callés et validés, il est nécessaire d'appréhender le fonctionnement hydrosédimentaire de l'estuaire à différentes échelles spatio-temporelles.

Certains grands domaines estuariens ont été l'objet d'études multi-proxies et multi échelles spatiales et temporelles pour montrer les rôles relatifs des différents facteurs de contrôle hydrodynamiques (BASSOULLET et al., 2000 ; revue dans DELOFFRE et al., 2006).

Dans le cas de l'estuaire de la Vilaine, il s'agit d'un domaine à dimensions réduites, par rapport aux environnements étudiés dans les travaux précédemment cités, et à régime fluvial contrôlé par un barrage bloquant la remontée des eaux salées à $8 \mathrm{~km}$ de l'embouchure. La construction de ce barrage a entraîné un comblement important en 30 ans, modifiant profondément la morphologie de l'estuaire et les pratiques des usagers (mytiliculture, civelles, coques, navigation). La gestion raisonnée des ressources de l'estuaire nécessite une compréhension approfondie du fonctionnement hydrodynamique et sédimentaire pour proposer des scénarios d'aménagements adaptés aux différentes contraintes du milieu. Ainsi, la modélisation hydrosédimentaire de l'estuaire a été initiée. Cette modélisation repose sur des données acquises spécifiquement pour cette étude et sur l'analyse des données disponibles.

La présente étude aborde la caractérisation du fonctionnement hydrosédimentaire de la Vilaine à partir de l'analyse (1) des données bathymétriques de 1820 à nos jours et (2) des suivis altimétriques (Altus) de la vasière intertidale septentrionale associés à différentes données caractérisant l'hydrodynamisme (marée, agitation de l'eau, vent, turbidité, débit fluvial), de novembre 2007 à avril 2008. L'objectif de cette approche est de définir l'impact d'événements hydrodynamiques sur l'évolution altimétrique d'un domaine intertidal afin d'alimenter un modèle hydrosédimentaire.

\section{L'estuaire de la Vilaine et le barrage d'Arzal}

La Vilaine (Figure 1), fleuve d'une longueur d'environ $220 \mathrm{~km}$ draine un bassin versant d'environ $10000 \mathrm{~km}^{2}$ et se termine par un estuaire s'ouvrant sur la Baie de Vilaine. Cette baie, d'une profondeur maximum de $30 \mathrm{~m}$ est partiellement protégée des houles du large par un arc de presqu'îles et d'îles. 
L'estuaire de la Vilaine, d'une orientation globale Est-Ouest est soumis aux actions de la houle, aux débits sortant du barrage et à un régime de marée méso à macrotidal (marnages de 2,5 $\mathrm{m}$ en mortes eaux et de plus de $5 \mathrm{~m}$ en vives eaux). Cet estuaire (Figure 1) est caractérisé à l'Est par une morphologie chenalisante de type méandriforme, l'estuaire interne, pour évoluer vers l'Ouest en une forme plus rectiligne qui s'évase vers l'embouchure, l'estuaire intermédiaire. La limite entre l'estuaire intermédiaire et la zone externe est marquée par un haut fond vaseux. Une forme de type delta sous-marin caractérise cette zone externe. La morphologie générale de cet estuaire indique qu'il s'agit d'un domaine intermédiaire entre un estuaire dominé par les vagues partiellement ouvert et un estuaire dominé par la marée selon DALRYMPLE et CHOI (2007).

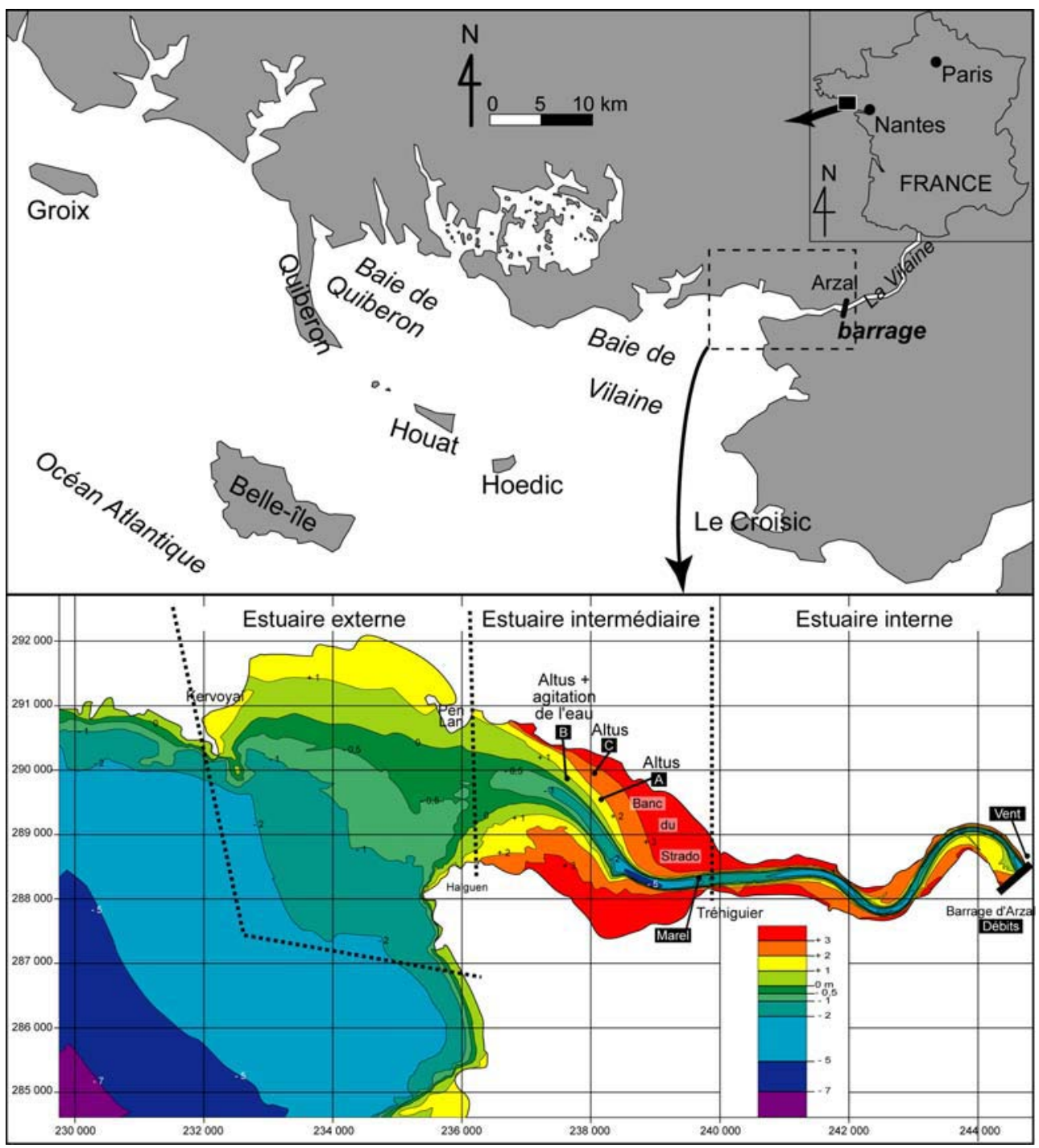

Figure 1. L'estuaire de la Vilaine: localisation, bathymétrie (2007) et plan de position des stations de mesures. 
Au cours des années 1960, la construction du barrage d'Arzal, situé à $8 \mathrm{~km}$ de l'embouchure, a permis de réduire les inondations des marais de Redon en empêchant les eaux saumâtres et chargées en particules fines de remonter. Les sédiments superficiels présents sur la zone d'étude sont des vases sableuses, avec une fraction inférieure à $45 \mu \mathrm{m}$ allant de 10 à $99 \%$ selon la saison (GOUBERT, 1997). Les vases sont remises en suspension par la houle et les vagues de tempête, puis elles sont transportées à marée montante vers l'estuaire (LCHF, 1960-1964).

L'installation de ce barrage a entraîné la réduction du volume oscillant, la diminution des vitesses de courant et ainsi provoqué (1) le déplacement de la zone de turbidité maximum vers l'aval (secteur intermédiaire) et (2) l'augmentation de la décantation des fractions fines dans ce secteur (LCHF, 1960-1964; MAILLOCHEAU, 1980 ; GOUBERT, 1997).

\section{Matériels et méthodes}

\subsection{Données bathymétriques de 1820 à 2007}

De 1820 à 2007, les données de douze relevés bathymétriques sont disponibles. Les relevés ont été acquis au plomb suiffé, puis au sondeur à $200 \mathrm{kHz}$ et $33 \mathrm{kHz}$. Les données ont été traitées sous MapInfo/Vertical Mapper (GOUBERT et MENIER, 2005), puis sous MIKE afin de réaliser des coupes sériées et le calcul des soldes sédimentaires.

\subsection{Suivis altimétriques et données connexes (Figure 1)}

Du 7 novembre 2007 au 4 avril 2008, trois systèmes Altus (JESTIN et al., 1998) ont mesuré les variations altimétriques de la vasière intertidale septentrionale et la hauteur de la tranche d'eau. Ce dispositif émet une onde à $2 \mathrm{MHz}$ qui permet d'estimer la distance entre le transducteur et l'interface eau/sédiment avec une précision de $\pm 2 \mathrm{~mm}$. Les Altus $\mathrm{A}$ et $\mathrm{C}$ ont enregistré ces données toutes les 2 minutes. L'Altus B a été programmé pour calculer les hauteurs significatives des vagues (une mesure toutes les 0,5 seconde pendant 4 min 40 , reprise 15 min plus tard). Toutes les 2 à 3 semaines, un suivi terrain était effectué pour décrire l'état de la vasière et décharger les données des Altus. Les trois Altus sont disposés en triangle $(500 \mathrm{~m}$ de côté) de manière à définir deux gradients : un gradient amont/aval (Altus A, C et B) et un gradient altimétrique du chenal vers la côte avec l'Altus B, le A et le C. Différentes données ont été utilisées pour caractériser l'état hydrodynamique de l'estuaire. Au barrage sont mesurés les volumes évacués par heure (exprimés en débit horaire en $\mathrm{m}^{3} / \mathrm{s}$ ) ainsi que la vitesse et la direction du vent, moyennées sur $10 \mathrm{~min}$. Au niveau de Tréhiguier, une bouée Marel mesure toutes les $10 \mathrm{~min}$ la turbidité en NTU. 


\section{Résultats et interprétations}

\subsection{Evolution de la bathymétrie de 1820 à 2007}

La comparaison des relevés de 1820 et 1960 montre que selon les secteurs, l'estuaire est caractérisée par un exhaussement global des fonds de 1 à $4 \mathrm{~m}$, avec l'exhaussement maximal de $4 \mathrm{~m}$ observé au niveau de la vasière septentrionale (Banc du Strado). Sur la période, de 1960 à 2003, les calculs des soldes sédimentaires, entre chaque suivi bathymétrique, révèle que dans la zone estuarienne interne et intermédiaire (Figure 1), 16 millions de $\mathrm{m}^{3}$ ont comblé l'estuaire en environ 30 ans (Figure 2). Depuis le début des années 1990, entre 1 et 2 millions de $\mathrm{m}^{3}$ de sédiments sont érodés/déposés respectivement au niveau principalement du chenal/des vasières, en fonction des périodes de crues (1995), de tempêtes (1999 et 2000) ou de calme (de 2001 à 2003). En ce qui concerne le Banc du Strado, il est caractérisé par un exhaussement de 4 à $6 \mathrm{~m}$ entre le relevé de 1960 et celui de 1992. Entre chaque relevé de 1992 à 2007, le Banc du Strado montre soit une morphologie stable lors des épisodes globalement érosifs (crues et tempêtes), soit des exhaussements de $40 \mathrm{~cm}$ maximum lors des épisodes calmes.

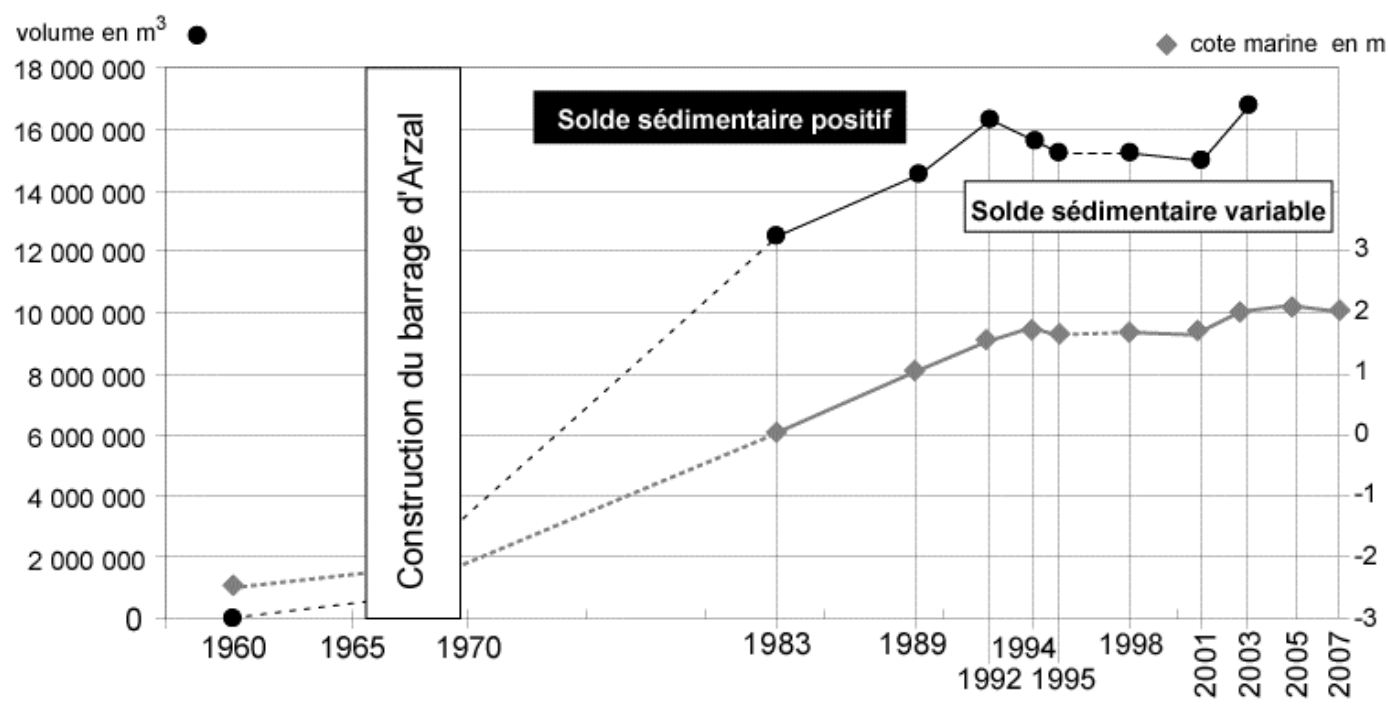

Figure 2. Evolution des soldes sédimentaires calculés pour les secteurs interne et intermédiaire (d'après ERAMM, 1995 et GOUBERT et MENIER, 2005) et évolution de l'altitude en cote marine du Banc du Strado (secteur des Altus).

Ces tendances semblent indiquées que globalement l'estuaire a atteint un équilibre morphodynamique depuis 1990 environ, avec des phases d'érosion dues aux épisodes de crues et de tempêtes. En revanche, le secteur du Banc du Strado, siège d'une accrétion à un rythme variable, montre qu'il y a encore de l'espace disponible dans l'estuaire de la Vilaine et qu'il est important de caractériser les variations morphologiques en fonction des conditions hydrodynamiques. 


\subsection{Evolution de la vasière intertidale septentrionale (Banc du Strado) du 7 novembre 2007 au 4 avril 2008 : altimétrie et état de surface}

Après un état relativement stable (état 0 , Figure 3), les évolutions altimétriques de fin novembre 2007 à début avril 2008 (Figure 3a) montrent de nombreuses phases d'érosion et de dépôt. Les phases d'érosion s'observent lorsque les coups de vents d'Ouest de vitesse supérieure à $10 \mathrm{~m} / \mathrm{s}$ provoquent des pics de Hs. En revanche, quelle que soit la vitesse du vent, lorsque celui-ci vient de l'Est, le plan d'eau est calme et les matières en suspension se décantent et les vasières engraissent.

La durée et l'ampleur de ces deux cas, érosion ou dépôt, ainsi que les occurrences de pics de turbidité à Tréhiguier vont dépendre des conditions de marée et de débit. Après $8 \mathrm{~h}$ de forts vents d'Ouest, le paroxysme de la tempête du 2 décembre a eu lieu à marée descendante n'entraînant qu'une faible augmentation de la turbidité à Tréhiguier. En revanche, la tempête du 9 décembre a atteint son maximum à marée montante. Cette conjonction a alors entraîné un important pic de turbidité à Tréhiguier, suite à cet apport aval de matière en suspension. Dans les jours suivants, l'augmentation des débits a provoqué une forte turbidité à Tréhiguier en raison, vraisemblablement, de la remise en suspension des sédiments dans la partie interne de l'estuaire. Après ce premier épisode d'érosion, les données fragmentaires de l'Altus du bord du chenal montrent globalement une tendance à l'engraissement, avec un dépôt d'environ $20 \mathrm{~cm}$ en 5 mois.

Les deux autres Altus ont évolué de manière similaire ou opposée, selon leur localisation géographique et les conditions hydrodynamiques. Après le 10 décembre, une phase de dépôt débute par quelques jours de relative stabilité, sans décantation, vraisemblablement due aux forts débits du barrage (flèche noire). Par la suite, les débits diminuant et les coefficients de marée descendant, les matières remises en suspension début décembre commencent à décanter. Cette décantation est interrompue fin décembre par deux coups de vent d'Ouest et du Sud provoquant un épisode érosif qui va modeler la surface de la vasière en seillons/banquettes (SB) parallèles, d'une largeur de 30 à $50 \mathrm{~cm}$, d'une profondeur de 10 à $20 \mathrm{~cm}$ et orientés perpendiculairement au courbes bathymétriques (GOULEAU et al., 2000 ; WHITEHOUSE et al., 2000). A l'issue de cet épisode érosif, l'Altus A était au dessus d'un seillon et l'Altus C au dessus d'une banquette. A partir de janvier 2008, une succession de coups de vent de secteur Sud à Ouest, se traduit par l'alternance de dépôt et d'érosion de vase très fluide dans les seillons (flèches noires, Altus A) alors que les banquettes vont être légèrement rabotées (Altus $C$ ). Début février, la surface de la vasière est à nouveau plane. Parallèlement, le secteur de l'Atlus B (bord du chenal non atteint par le système SB) a enregistré un dépôt de vase fluide de $6 \mathrm{~cm}$ d'épaisseur. 


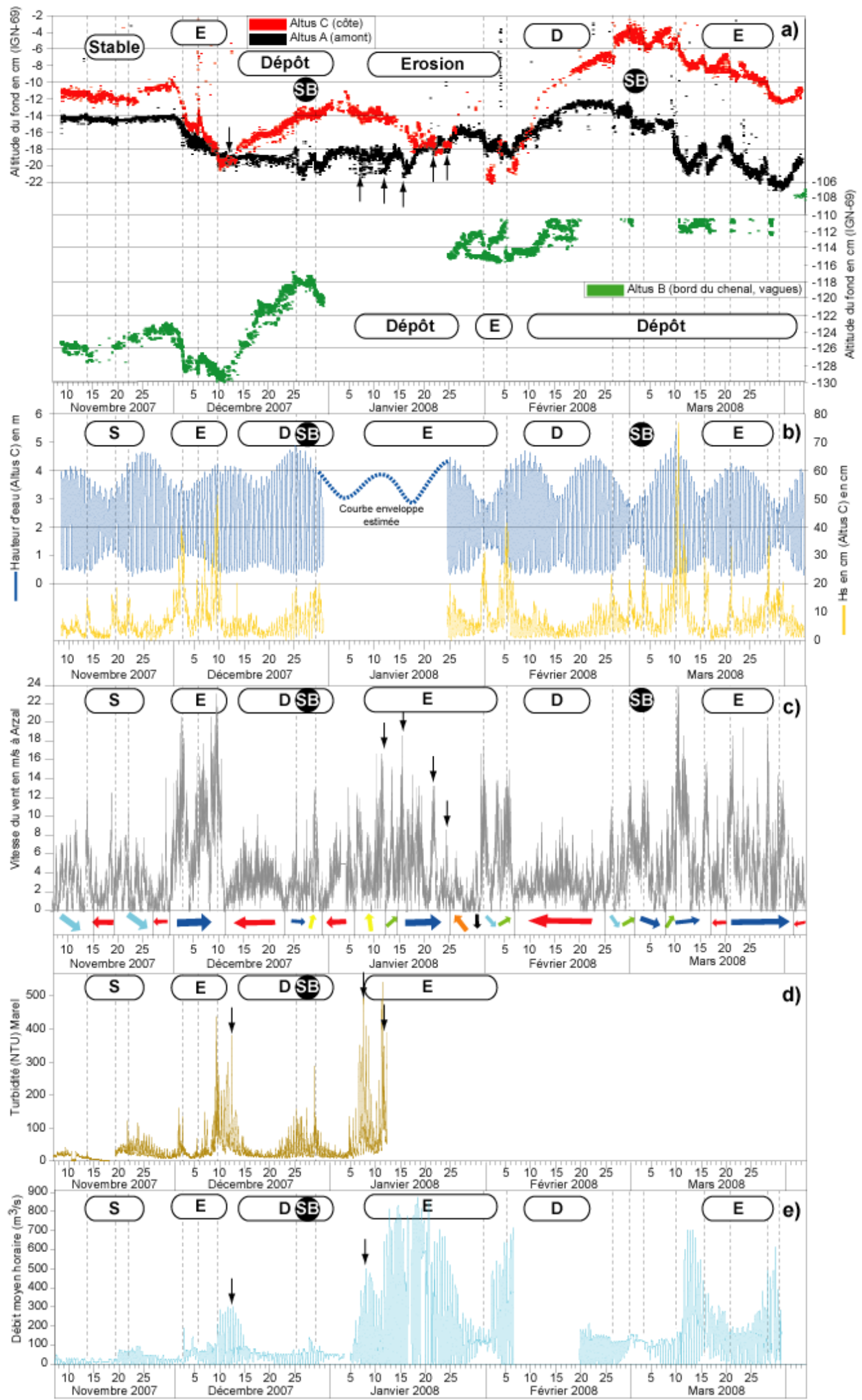

Figure 3. Suivis hydrosédimentaires de l'estuaire de la Vilaine du 7 novembre 2007 au 4 avril 2008. (SB : seillons et banquettes) 
Cette phase, caractérisée par des vents soutenus et des forts débits, se termine par un coup de vent double début février qui affecte principalement le secteur de l'Altus B en raison de l'érodabilité plus grande de la vase fluide fraîchement déposée. Suite à cette phase, les MES vont se décanter sur l'ensemble de la zone au cours du mois de février (déchet).

Du 28 février au 9 mars, les structures SB réapparaissent. Il semble que cette deuxième occurrence de SB soit la réactivation de la structure antérieure en érodant tout d'abord la vase plus fluide située au niveau des anciens seillons, puis en surcreusant ces seillons. La tempête du 10 mars $(25 \mathrm{~m} / \mathrm{s})$ a érodé fortement la vasière $(6$ à $8 \mathrm{~cm})$ et effacé le système SB. Enfin, du 13 mars au 4 avril, le même climat hydrodynamique que celui du mois de janvier a régné sur l'estuaire : succession de coups de vent de Nord-Ouest à Sud-Ouest, de vitesse moyenne. L'altitude globale de la vasière diminue, avec une succession de cycles : dépôt de 1 à $2 \mathrm{~cm}$ de vase fluide pendant les jours calmes puis érosion 2 à $3 \mathrm{~cm}$ au cours des coups de vent. A la différence du mois de janvier, cet épisode se déroule sur une vasière plane, alors qu'en janvier il s'agissait d'un système SB.

\section{Discussion}

Le suivi altimétrique par la méthode Altus de la vasière intertidale septentrionale, associé à l'étude de l'état de surface, a permis la quantification des dépôts et des érosions au cours d'une période hivernale riche en tempêtes et coups de vent d'orientations et de vitesses variables. Ainsi, sur l'ensemble de la période étudiée, l'ensemble de la vasière a été érodé de 6 à $11 \mathrm{~cm}$ lors de la première tempête de décembre et de 4 à $6 \mathrm{~cm}$ lors de la tempête de mars. Pour les taux de sédimentation, ils ont atteint $26 \mathrm{~cm}$ en quatre mois pour l'Altus $\mathrm{B}$ et $16 \mathrm{~cm}$ au cours du mois de février pour l'Altus C (côte). Ces taux sont supérieurs à ceux observés à Marennes-Oléron (BASSOULLET et al., 2000). En revanche, ils sont comparables à ceux observés en Seine (DELOFFRE et al., 2006), alors que la Seine a des dimensions géographiques et hydrodynamiques deux à dix fois supérieurs à ceux de l'estuaire de la Vilaine et des sédiments plus sableux.

A partir de l'approche méthodologique particulière (3 Altus disposés en triangle de $500 \mathrm{~m}$ de côté et descriptions régulières de l'état de surface de la vasière), il a été possible de préciser les relations entre le vent, les hauteurs significatives des vagues et la dynamique sédimentaire. A chaque coup de vent d'Ouest, quelles que soient les autres conditions, l'agitation du plan d'eau entraîne une phase d'érosion nettement visible au niveau de la partie haute de la vasière : la hauteur et l'ampleur de la zone concernée dépendant de la vitesse du vent. Dans ce contexte, les autres conditions (débit et marée) vont contrôler le devenir des matières 
remises en suspension. Si l'on est en phase de déchet, les MES vont se décanter les jours suivants. Si l'on est en phase de revif, les MES vont décanter moins rapidement ou être transportées hors de la vasière. Si les débits sont importants, les MES seront expulsées de l'estuaire. A l'opposé, lors d'épisodes de vents d'Est, la tendance est à l'engraissement de l'ensemble de la vasière (Altus A et C).

L'observation du fonctionnement de la vasière septentrionale indique que cette zone possède encore de l'espace disponible à la limite du chenal de navigation.

Ainsi, à l'échelle globale des relevés bathymétriques, l'estuaire de la Vilaine semble avoir atteint un certain équilibre; en revanche, à l'échelle des caractéristiques morphologiques du système vasière/chenal, il semble que la tendance soit toujours à l'aggradation conduisant à la progradation de la vasière nord vers le centre du chenal de navigation. Ainsi, à terme, la largeur et la profondeur du chenal de navigation peuvent encore se réduire dans la zone de l'embouchure, entraînant l'accroissement des difficultés de navigation.

\section{Conclusion}

L'estuaire de la Vilaine présente une dynamique sédimentaire dont les caractéristiques sont principalement contrôlées par la présence du barrage d'Aral (construit au milieu des années 1960) à $8 \mathrm{~km}$ de son embouchure. En modifiant la géométrie et l'hydrodynamisme de l'estuaire, l'implantation et les modalités de fonctionnement du barrage ont provoqué une augmentation très forte de la vitesse de comblement de l'estuaire jusqu'au début des années 1990. Depuis, la tendance semble toujours être à l'engraissement, mais avec une vitesse moins grande.

Grâce aux données altimétriques et hydrologiques, l'identification des conditions hydrodynamiques entraînant des phases d'érosion ou de dépôt va permettre de caler et de valider le modèle hydrosédimentaire de la Vilaine dans le cas de scénarios hydrodynamiques particuliers (conjonctions vent/Hs/débit/marée, vasière à surface plane, seillons/banquettes, couche de vase fluide). Parallèlement, ces informations peuvent être utiles aux gestionnaires du barrage d'Arzal afin de mieux prendre en compte les conséquences des modalités d'ouverture du barrage sur la dynamique hydrosédimentaire de la partie estuarienne de la Vilaine.

\section{Remerciements}

Ce travail a été effectué dans le cadre d'une convention d'étude entre l'Institution d'Aménagement de la Vilaine, le Danish Hydraulic Institut et l'Université de Bretagne Sud. Nous remercions l'Ifremer et l'IAV pour les données de la bouée Marel, et surtout Hervé Jestin, Philippe Bassoullet et Pierre Le Hir pour leurs précieux conseils sur les Altus et pour la mise à disposition de ces dispositifs. 


\section{Références bibliographiques}

1. BASSOULlET P., LE HIR P., GOULEAU D., ROBERT S. (2000). Sediment transport over an intertidal mudflat: field investigations and estimation of fluxes within the "Baie de Marenngres-Oléron" (France). Continental Shelf Research, 20, 12-13, 1635-1653.

2. DALRYMPLE R.W., CHOI K. (2007). Morphologic and facies trends through the fluvial-marine transition in tide-dominated depositional systems : a schematic framework for environmental and sequence-stratigraphic interpratation. Earth-Science Reviews, 81, 135-174.

3. DELOFFRE J., LAFITE R., LESUEUR P., VERNEY R., LESOURD S., CUVILLIEZ A., TAYLOR J. (2006). Controling factors of rythmic sedimentation processes on an intertidal estuarine mudflat. Role of the turbidity maximum in the macrotidal Seine estuary, France. Marine Geology, 235, 151-164.

4. ERAMM-RIVAGES, 1995. Etude de l'envasement de l'estuaire de la Vilaine. Rapport Institution d'Aménagement de la Vilaine, 110 p.

5. GOUBERT E. (1997). Les Elphidium excavatum, foraminifères benthiques, vivant en baie de Vilaine d'octobre 1992 à septembre 1996. Thèse de doctorat Université de Nantes, 2 vol., 310 p.

6. GOUBERT E., MENIER D. (2005). Evolution morphosédimentologique de l'estuaire de la Vilaine de 1960 à 2003 : Valorisation des campagnes bathymetriques. Rapport Institution d'Aménagement de la Vilaine, 104 p.

7. GOULEAU D., JOUANNEAU J.M., WEBER O., SAURIAU P.G. (2000). Short- and long-term sedimentation on Montportail-Brouage intertidal mudflat, Marennes-Oléron Bay (France). Continental Shelf Research, 20, 1513-1530.

8. JESTIN H., BASSOULLET P., LE HIR P., L'YAVANC J., DEGRES Y. (1998). Development of ALTUS, a high Frequency acoustic submersible recording altimeter to accurately monitor bed elevation and quantify deposition or erosion of sediments. Conference Oceans'98, vol.1/3, 189-194.

9. LCHF (1960-1964). Mission d'étude de la Vilaine - Barrage d'Arzal : Etudes sédimentologiques et hydrauliques. Archives Institution d'Aménagement de la Vilaine.

10. MAILlOCHEAU F. (1980). L'envasement de l'estuaire de la Vilaine en aval du barrage d'Arzal. DEA de Géologie Marine, Université de Nantes, 65 p.

11. WHITEHOUSE R.J.S., BASSOULLET P., DYER K.R., MITCHENER H.J., ROBERTS W. (2000). The influence of bedforms on flows and sediment transport over intertidal mudflats. Continental Shelf Research, 20, 1099-1124. 